\title{
Identificación de ecto y endoparásitos en palomas domésticas (Columba livia) del área urbana de Villavicencio, Meta, Colombia
}

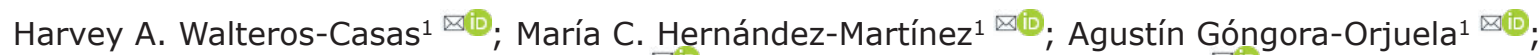
Jorge L. Parra-Arango ${ }^{\otimes(\mathbb{0}}$; Jenny J. Chaparro-Gutiérrez ${ }^{2 *} \bowtie \mathbb{O}^{\circ}$.

${ }^{1}$ Universidad de los Llanos. Escuela de Ciencias Animales. Medicina Veterinaria y Zootecnia.

¿Universidad de Antioquia UdeA, Facultad de Ciencias Agrarias. Grupo de Investigación CIBAV, Medellín Antioquia.

*Correspondencia: jenny.chaparro@udea.edu.co

Recibido: Octubre 2020; Aceptado: Marzo 2021; Publicado: Junio 2021.

\section{RESUMEN}

Objetivo. Determinar la presencia de parásitos internos y externos en palomas comunes (Columba livia) del área urbana de la ciudad de Villavicencio, Meta, durante la transición verano a invierno del año 2017. Materiales y Métodos. Se capturaron 72 palomas de tres comunas de la ciudad y sometidas a inspección visual para parásitos externos, a la vez se obtuvo muestras de material fecal para análisis coprológico mediante el método de Sheather's. Se realizaron frotis con las muestras de sangre y tinción con Giemsa para agentes hemotrópicos. Resultados. En el $100 \%$ de las muestras se encontraron dos especies de parásitos externos: piojos malófagos (Columbicola columbae) y mosca de paloma (Pseudolynchia canariensis). Dentro de los parásitos internos se encontraron dos protozoarios: Haemoproteus spp. 26.5\% (9/34) en frotis sanguíneo y Eimeria spp. 36\% (26/72) en heces, seguido de los nematodos: Ascaridia spp. $4.2 \%$ (3/72) y Capillaria spp. $13.8 \%(10 / 72)$ y cestodos: Raillietina spp. 1.38\% (1/72). No se encontró asociación del parasitismo con el sexo de la paloma o la comuna de muestreo. Conclusiones. La presencia de parásitos internos fue baja, excepto para Eimeria spp., estos datos representan información importante del riesgo potencial para la salud animal y humana, especialmente para poblaciones comerciales de aves y la avifauna nativa. Estos resultados indican que se requieren programas sanitarios y de control en las poblaciones de palomas de la ciudad.

Palabras clave: Parasitosis; Columbicola columbae; parásitos (Fuente: DeCs).

\section{ABSTRACT}

Objetive. Determine the presence of internal and external parasites in common pigeons (Columba livia) in the urban area of the city of Villavicencio, Meta, during the transition from summer to winter in 2017. Materials and Methods. 72 pigeons from three communes of the city were captured and subjected to visual inspection for external parasites, at the same time, samples of fecal material were obtained for stool analysis using the Sheather's method. Blood samples were smeared and Giemsa stained for hemotropic agents. Results. Two species of external parasites were found in $100 \%$ of

Como citar (Vancouver).

Walteros-Casas HA, Hernández-Martínez MC, Góngora-Orjuela A, Parra Arango JL, Chaparro-Gutiérrez JJ. Identificación de ecto y endoparásitos en palomas domésticas (Columba livia) del área urbana de Villavicencio, Meta, Colombia. Rev MVZ Córdoba. 2021; 26(3):e2157. https://doi.org/10.21897/rmvz.2157

CEl (los) autor (es), Revista MVZ Córdoba 2021. Este artículo se distribuye bajo los términos de la licencia internacional Creative Commons Attribution CC) $\$ 4.0$ (https://creativecommons.org/licenses/by-nc-sa/4.0/), que permite a otros distribuir, remezclar, retocar, y crear a partir de su obra de modo no BY NC SA comercial, siempre y cuando den crédito y licencien sus nuevas creaciones bajo las mismas condiciones. 
the samples: Mallophaga lice (Columbicola columbae) and pigeon fly (Pseudolynchia canariensis). Within the internal parasites, two protozoa were found: Haemoproteus spp. (9/34) in blood smear and Eimeria spp. (26/72) in feces, followed by nematodes: Ascaridia spp. (3/72) and Capillaria spp. (10/72) and cestodes: Raillietina spp. (1/72). No association of parasitism was found with the sex of the pigeon or the sampling commune. Conclusions. The presence of internal parasites was low, except for Eimeria spp., these data represent important information on the potential risk for animal and human health, especially for commercial populations of birds and native avifauna. These results indicate that sanitary and control programs are required in the pigeon populations of the city.

Keywords: Columba livia; Columbicola columbae; parasitism (Source: DeCs).

\section{INTRODUCCIÓN}

Las palomas domésticas (Columba livia domestica) son aves ferales urbanas adaptadas a vivir en diversos nichos, se encuentra distribuida en todos los continentes excepto en la Antártida (1). De acuerdo a Del Hoyo et al (2), esta especie se originó en una extensa área de Eurasia y Africa (2).

Un análisis de riesgo para Colombia como especie invasora, la cataloga como "de alto riesgo", por lo que debería estar sujeta a control y de acciones de manejo, educación ambiental y legislación específica para la prevención y mitigación del riesgo $(3,4)$. A pesar de lo anterior, se ha hecho poco para evitar el aumento de la población en gran parte de las áreas urbanas y rurales del país, convirtiéndose en una verdadera plaga en todo el territorio nacional.

Son cuantiosas las pérdidas económicas atribuidas a las palomas, las cuales se ven representadas en daños a los alimentos almacenados, deterioro de edificios e instalaciones, competencia por espacio y alimento con la avifauna nativa $(5,6)$ Sin embargo, los efectos más graves están representados en la potencial fuente de trasmisión de agentes zoonóticos que afectan la salud pública como, histoplasmosis, ornitosis, salmonelosis, cryptococcosis, campilobacteriosis y clamidiosis $(7,8,9,10)$. Varias enfermedades afectan la salud de las palomas, siendo el parasitismo interno y externo una de las más frecuentemente repor tadas $(11,12,13)$. Las palomas, además, pueden servir de reservorio de infecciones parasitarias, bacterianas y virales para otras aves $(14,15)$. Debido al aumento de la población de palomas en la ciudad de Villavicencio y el estrecho contacto con los humanos, explotaciones comerciales de otras especies de aves y la avifauna nativa, el objetivo de este estudio fue identificar los principales parásitos internos y externos que afectan a esta especie en tres comunas del área urbana.

\section{MATERIALES Y MÉTODOS}

Ubicación y tamaño de la muestra. Se realizó un estudio epidemiológico transversal en las comunas 2, 3 y 4 de la ciudad de Villavicencio, Meta, que corresponden a las más antiguas, desde el punto de vista arquitectónico y las de mayor población de palomas (Figura 1). El diseño de la muestra fue de tipo probabilístico por Epidat 3.1, tomando como base un universo de 14.718 palomas, de acuerdo con datos no publicados de González et al (comunicación personal) con una fracción de muestreo del $70 \%$.

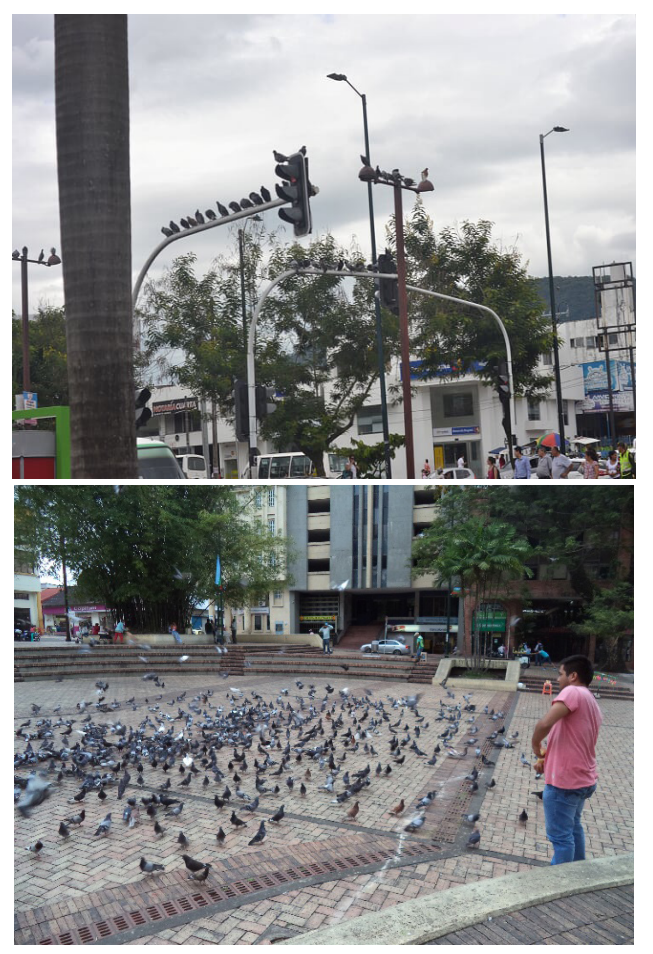

Figura 1. Presencia de palomas en sitio público, comuna 2.

Para determinar el tamaño de la población de palomas comunes (Columba livia) ubicadas en vías públicas del área urbana de Villavicencio, se tuvo en cuenta la información obtenida por 
González et al. (Comunicación personal) de una población estimada de 14.718 aves.

De este reporte, solo se tuvieron en cuenta las comunas con mayor densidad poblacional de palomas, siendo las comunas 2,3 y 4 , las que cumplen esta característica, con una población de 10.343 palomas, $70.3 \%$ del total poblacional. Se hace el supuesto que el 10\% (de acuerdo al estudio de González et al, comunicación personal) ( $p$ ) de la población de palomas tienen alguna especie de parásito y un $90 \%$ no los tengan (q): Para determinar el tamaño de muestra, se empleó la siguiente formula con una probabilidad de éxito del $5 \%$, una confianza del $95 \%$ y una exactitud del $5 \%$ :

$$
n_{0}=\frac{Z^{2} * p * q * N}{(N-1) * e^{2}+Z^{2} * p * q}
$$

Siendo:

$\mathrm{n}$ = Tamaño estimado de la muestra

$Z$ = Valor de la distribución normal estándar

$\mathrm{N}$ = Tamaño de la población

$\mathrm{p}=$ Proporción estimada

$\mathrm{q}=1-\mathrm{P}$

$\mathrm{e}=$ Error de muestreo

Reemplazando:

$$
n_{0}=\frac{1,96^{2} * 0,05 * 0,95 * 14718}{(14718-1) * 0,05^{2}+1,96^{2} * 0,05 * 0,95}=72
$$

Estas 72 palomas se capturaron en las tres comunas, en un solo período climático, durante la transición verano e invierno (Tabla 1, Figura 2).

Tabla 1. Distribución del muestreo.

\begin{tabular}{cccc}
\hline Comuna & $\begin{array}{c}\text { Población de } \\
\text { palomas }\end{array}$ & $\begin{array}{c}\text { \% Total de } \\
\text { población }\end{array}$ & $\begin{array}{c}\text { Tamaño de } \\
\text { muestra }\end{array}$ \\
\hline 2 & 6.328 & $61,18 \%$ & 44 \\
3 & 2.205 & $21,32 \%$ & 15 \\
4 & 1.810 & $17,50 \%$ & 13 \\
\hline Total & $\mathbf{1 0 . 3 4 3}$ & $\mathbf{1 0 0} \%$ & $\mathbf{7 2}$ \\
\hline
\end{tabular}

Captura y necropsia. Las palomas fueron capturadas en el período de transición verano e invierno del año 2017 abril a junio, empleando una malla de nylon; se trasladaron en jaulas hasta el Laboratorio de Histopatología de la Universidad de los Llanos, en donde fueron sacrificadas con ketamina al $10 \%$, vía IM, a una dosis de 40 mg/ $\mathrm{kg}$. Se identificó el sexo por observación directa de las gónadas Durante la necropsia se hizo una revisión minuciosa del plumaje, colectando especímenes de ectoparásitos visibles en plumas y piel. La identificación de los ectoparásitos se realizó bajo estereo microscopio (Marca Nixon, modelo SMZ 445), empleando las claves de Clayton y Price (16), Aktison y Hunter (17) y Graciolli y Carvalho (18).

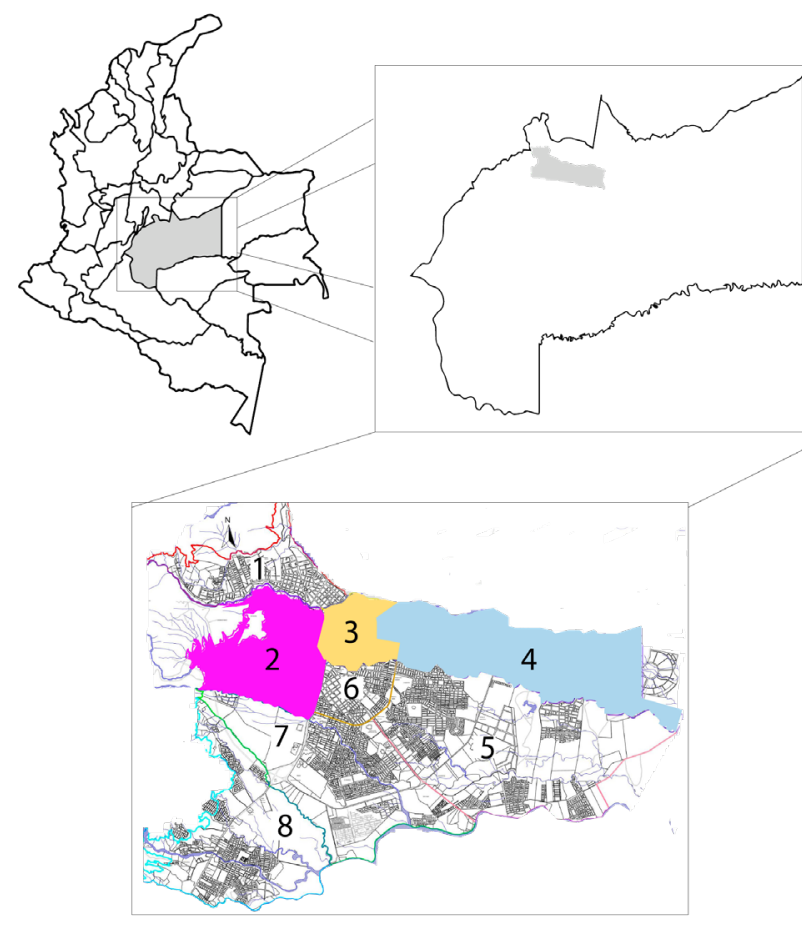

Figura 2. Mapa de Colombia, donde se ubica el departamento del Meta y plano del municipio de Villavicencio, que señala los lugares de muestreo comunas 2, 3 y 4.

Recolección de muestras. Antes de la eutanasia se extrajo sangre por punción de la vena braquial y se realizaron frotis sanguíneos por gota gruesa en láminas portaobjetos, para la identificación de hemoparásitos mediante la tinción de Giemsa. Se recolectaron $5 \mathrm{~g}$ de heces por ave de las porciones del intestino delgado e intestino grueso y fueron puestas en frascos de vidrio estériles y refrigerados $\left(4-8^{\circ} \mathrm{C}\right)$ hasta su procesamiento.

Frotis directo. Se hizo observación directa al microscopio a partir de un frotis de materia fecal, diluida en solución salina fisiológica al 0.9\% y en Lugol parasitológico, en la búsqueda de trofozoítos, quistes, huevos y ooquistes de parásitos intestinales. 
Técnica de concentración. Las muestras de materia fecal fueron procesadas por el método cualitativo de flotación en solución de Sheather, se tomó un gramo de materia fecal y $15 \mathrm{~mL}$ de solución saturada de azúcar con una densidad de 1.27 , se llenó el tubo hasta formar un menisco, sobre el cual se dispuso una laminilla cubreobjeto se dejó en reposo por 10 min y se procedió a colocar la laminilla cubreobjeto sobre una lámina portaobjeto y se observa al microscopio binocular en aumentos 10X, 20X, 40X y 100X. (Zeiss-Primo Star, Göttingen, Germany) (19).

Esporulación de coccidias. Las muestras positivas para coccidias (mínimo 5 coccidias por campo) se sometieron al proceso de esporulación en una solución de $2.5 \%(\mathrm{w} / \mathrm{v})$ de dicromato de potasio $\left(\mathrm{K}_{2} \mathrm{Cr}_{2} \mathrm{O}_{7}\right)$, las heces se mezclaron bien en cajas de Petri y se mantuvieron a temperatura ambiente en oscuridad y aireación para facilitar la esporulación. Las especies de coccidias se determinaron basados en las características morfológicas de los ooquistes y su tiempo de esporulación (20).

Tinción de Giemsa. se realizó frotis sanguíneo a partir de las muestras de sangre fresca, se hizo una fijación previa de las placas con etanol, posterior fueron cubiertas con el colorante a una dilución 1:10 por 12 minutos, se lavó con abundante agua y se observó al microscopio en el objetivo de 100X en busca de hemoparásitos. La identificación de parásitos sanguíneos se hizo siguiendo la técnica de Soulsby (21).

Análisis estadístico. Los resultados se analizaron por prueba exacta de Fisher y prueba de Chi cuadrado $\left(X^{2}\right)$ a una probabilidad del $95 \%$ $(a=0,05)$ relacionando la especie parasitaria, el sexo de la paloma y la comuna. Los datos se analizaron con el programa estadístico SPSS versión 23.0 para Windows y Epidat 3.1.

Aspectos éticos. Este proyecto fue aprobado por el Comité de Bioética de la Facultad de Ciencias Agropecuarias y Recursos Naturales de la Universidad de los Llanos, según Acta 003 de 18 abril de 2017.

\section{RESULTADOS}

De las 72 palomas capturadas, 19 fueron hembras y 53 machos. En el $100 \%$ de las palomas, se evidenció la presencia de dos tipos de parásitos externos: piojos malófagos (Columbicola columbae) y la mosca de la paloma (Pseudolynchia canariensis) (Figura 3); los primeros se encontraron principalmente en plumas remígeas, zona abdominal y pectoral, mientras las segundas no tenían una distribución diferenciada en el cuerpo.

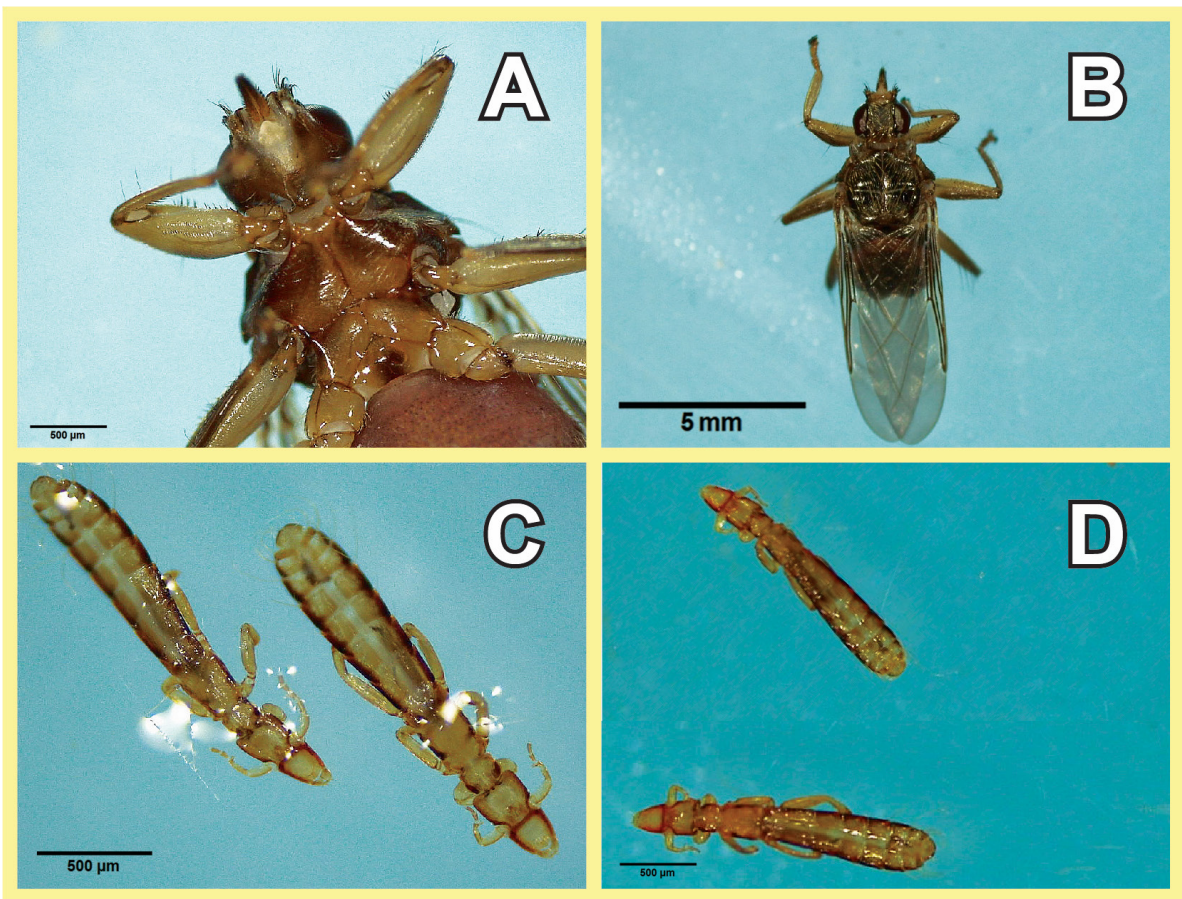

Figura 3. Ectoparásitos detectados en aves de la Familia Columbidae. (A y B) Mosca-Piojo de la paloma (Pseudolynchia canariensis); (C y D) Piojos malófagos (Columbicola columbae). 
Al examen macroscópico del tracto intestinal, se colectaron parásitos adultos en $58.33 \%$ (42/72) de las palomas, de los cuales $43.05 \%$ $(31 / 72)$ eran parásitos nematodos, $9.72 \%$ (7/72) parásitos segmentados compatibles con cestodos y en $6.94 \%$ (5/72) aves se encontró una asociación múltiple de nematodos y cestodos (Figura 4).

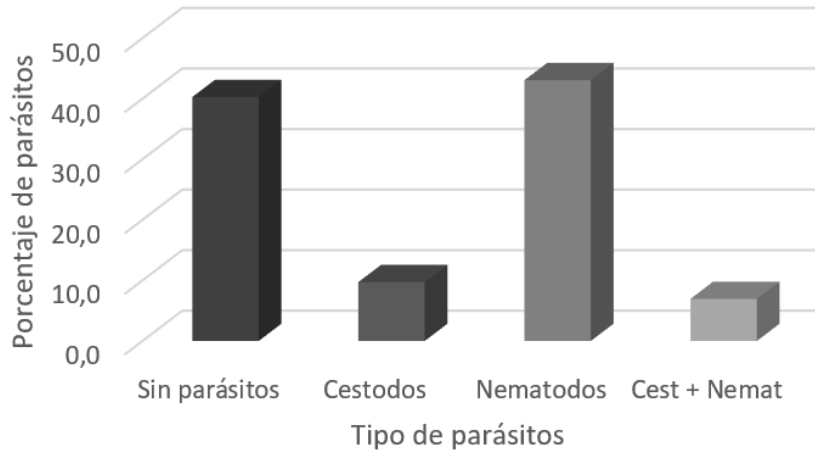

Figura 4. Recuento de parásitos adultos en intestino de palomas de Villavicencio

Se encontraron huevos de parásitos en muestras de heces de 30 palomas clasificadas de acuerdo con el tipo de huevos en: Tipo 1: un solo tipo de huevo; tipo 2: dos tipos de huevos; tipo 3: tres tipos de huevos (Figura 5).

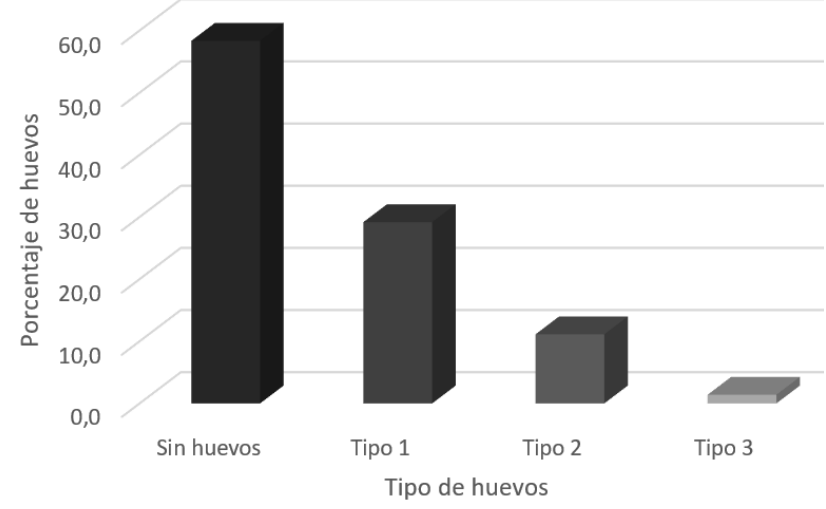

Figura 5. Recuento de tipos de huevos de parásitos en heces de palomas de Villavicencio.

El frotis directo de materia fecal del intestino delgado fue negativo en 69/72 de las muestras, encontrando solo una muestra con Ascaridia spp. y dos con Capillaria spp. En intestino grueso el $78 \%(56 / 72)$ de las muestras fueron negativas, identificando $1.39 \%(1 / 72)$ de Ascaridia spp., $2.8 \%(2 / 72)$ de Capillaria spp. y $16.67 \%$ (12/72) de Eimeria spp, con $1.4 \%(1 / 72)$ de muestras de Capillaria spp. y Eimeria spp. (Figura 6- Tabla 2).

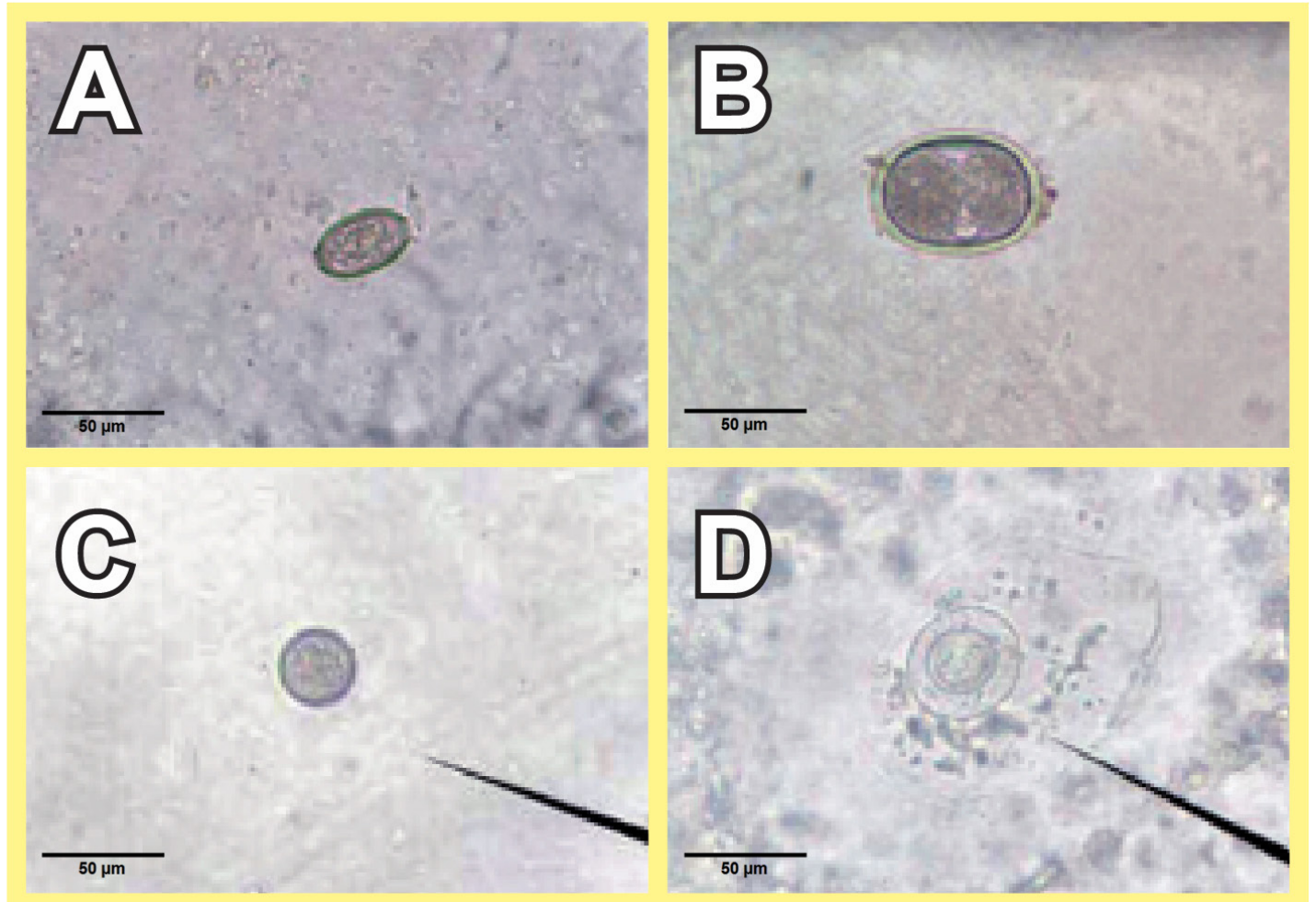

Figura 6. Microfotografías de endoparásitos detectados en aves de la Familia Columbidae. (A) huevo de Capillaria spp; (B) huevo de Ascaridia spp; (C) Ooquiste de coccidia (D) huevo de Raillietina spp. 
Walteros-Casas et al - Parásitos en palomas domésticas (Columba livia) de Colombia

Tabla 2. Número y porcentaje de parásitos encontrados por frotis directo de intestino delgado y grueso en palomas de Villavicencio.

\begin{tabular}{cccccc}
\hline Parásito & Intestino delgado & \% poblacional & Intestino grueso & \% poblacional & Total positivos \\
\hline Ascaridia spp. & $1 / 72$ & $1.39 \%$ & $1 / 72$ & $1.39 \%$ & $2.8 \%(2 / 72)$ \\
Capillaria spp. & $2 / 72$ & $2.78 \%$ & $2 / 72$ & $2.78 \%$ & $5.6 \%(4 / 72)$ \\
Eimeria spp & $0 / 72$ & $0 \%$ & $12 / 72$ & $16.67 \%$ & $16.67(12 / 72)$ \\
Eim. + Capil. & $0 / 72$ & $0 \%$ & $1 / 72$ & $1.39 \%$ & $1.39 \%(1 / 72)$ \\
Negativo & $69 / 72$ & $95.83 \%$ & $56 / 72$ & $77.77 \%$ & - \\
\hline Total & $\mathbf{7 2}$ & $\mathbf{1 0 0 \%}$ & $\mathbf{7 2}$ & $\mathbf{1 0 0}$ & $\mathbf{2 6 . 3 8 \% ( \mathbf { 1 9 / 7 2 } )}$ \\
\hline
\end{tabular}

Por la técnica de flotación 66/72 de las muestras de intestino delgado fueron negativas a huevos de parásitos intestinales, identificándose Raillietina spp., Ascaridia spp., Capillaria spp. y Eimeria spp. En intestino grueso hubo 48/72 de muestras negativas, encontrándose Capillaria spp., Eimeria spp. y 4/72 asociaciones de Eimeria más Capillaria (Tabla 3).
En general, la prevalencia de endoparásitos intestinales por el tipo de parásito fue del $33.3 \%(24 / 72)$ de Eimeria spp., $11.1 \%(8 / 72)$ de Capillaria spp., $1.39 \%(1 / 72)$ de Ascaridia spp., $1.39 \%(1 / 72)$ de Raillietina spp. y de protozoarios sanguíneos se encontraron $26.5 \%$ (9/34) de Haemoproteus spp. en frotis de sangre (Figura 7 - Tabla 4).

Tabla 3. Número y porcentaje de parásitos detectados por la técnica de Sheather en materia fecal de intestino delgado y grueso en palomas de Villavicencio.

\begin{tabular}{cccccc}
\hline Parásito & Intestino delgado & \% poblacional & Intestino grueso & \% poblacional & Total positivos \\
\hline Raillietina spp. & $1 / 72$ & $1.39 \%$ & $0 / 72$ & 0 & $1.39 \%(1 / 72)$ \\
Ascaridia spp & $1 / 72$ & $1.39 \%$ & $0 / 72$ & 0 & $1.39 \%(1 / 72)$ \\
Capillaria spp. & $1 / 72$ & $1.39 \%$ & $3 / 72$ & $4.17 \%$ & $5.55 \%(4 / 72)$ \\
Eimeria spp & $3 / 72$ & $4.17 \%$ & $17 / 72$ & $23.61 \%$ & $27.7 \%(20 / 72)$ \\
Eim. + Capil. & $0 / 72$ & 0 & $4 / 72$ & $5.56 \%$ & $5.55 \%(4 / 72)$ \\
Negativo & $66 / 72$ & $91.66 \%$ & $48 / 72$ & 66.66 & - \\
\hline Total & $\mathbf{7 2}$ & $\mathbf{1 0 0 \%}$ & $\mathbf{7 2}$ & $\mathbf{1 0 0} \%$ & $\mathbf{4 1 . 6 \% ( 3 0 / 7 2 )}$ \\
\hline
\end{tabular}

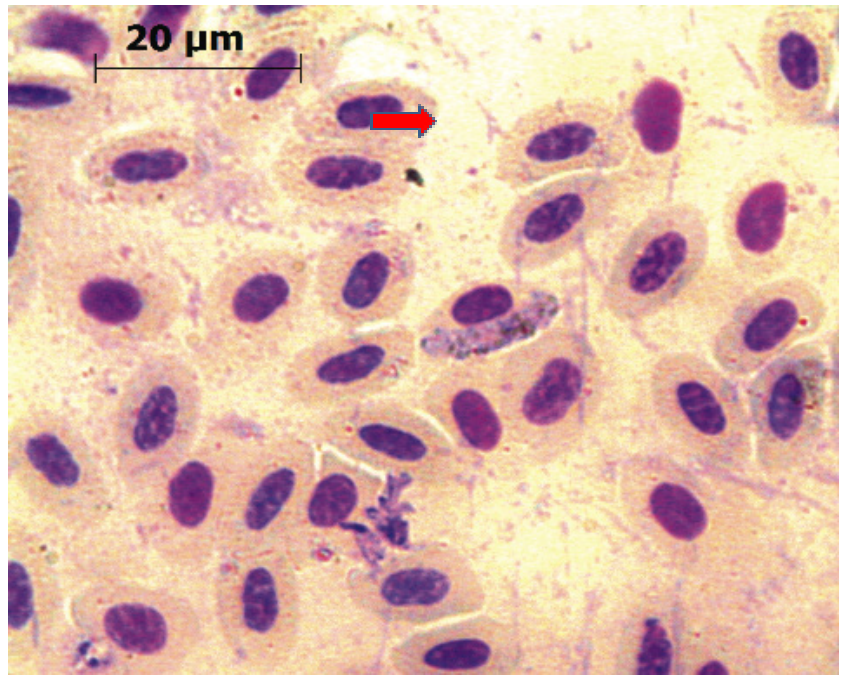

Figura 7. Gametocito de Haemoproteus columbae, ver flecha
Tabla 4. Prevalencia de parásitos en palomas estudiadas de tres comunas de Villavicencio.

\begin{tabular}{cccc}
\hline $\begin{array}{c}\text { Grupo } \\
\text { parasitario }\end{array}$ & Especie & n & $\begin{array}{c}\text { Porcentaje de } \\
\text { Prevalencia }\end{array}$ \\
\hline Cestodos & Raillietina spp. & $1 / 72$ & $2.04 \%$ \\
& Ascaridia spp & $3 / 72$ & $6.12 \%$ \\
Nematodos & Capillaria spp. & $10 / 72$ & $16.33 \%$ \\
$\begin{array}{c}\text { Protozoarios } \\
\text { intestinales }\end{array}$ & Eimeria spp. & $26 / 72$ & $65.31 \%$ \\
$\begin{array}{c}\text { Protozoarios } \\
\text { sanguíneos }\end{array}$ & Haemoproteus spp. & $9 / 34$ & $26.47 \%$ \\
\hline
\end{tabular}


Se relacionaron los parásitos intestinales y el sexo de las palomas empleando el análisis de frecuencia de tablas de contingencia correlacionando las variables categóricas, su significancia estadística se evaluó por prueba de Chi cuadrado $\left(X^{2}\right)$ con una confianza del 95\%; encontrándose que no existe relación entre la presencia de una especie parasitaria dada y el sexo de las palomas (Tabla 5).
Al relacionar los parásitos intestinales con las comunas muestreadas $(2,3$ y 4$)$, empleando el análisis de frecuencia de tablas de contingencia correlacionando las variables categóricas, se encontró que no existe relación entre éstas (Tabla 6).

Tabla 5. Análisis estadístico bivariado (Chi cuadrado) entre parásitos intestinales y sexo de las palomas.

\begin{tabular}{|c|c|c|c|c|c|c|}
\hline \multirow{2}{*}{ Parásito } & \multicolumn{2}{|c|}{ Sexo } & \multirow{2}{*}{$X^{2}$} & \multirow{2}{*}{$\mathbf{P}$} & \multirow{2}{*}{$\begin{array}{c}\text { Razón de } \\
\text { prevalencia }\end{array}$} & \multirow{2}{*}{$\begin{array}{c}\text { Intervalo de } \\
\text { confianza } \\
\end{array}$} \\
\hline & Hembra & Macho & & & & \\
\hline Ascaridia spp. & 2 & 1 & 2.614 & 0.106 & 5.579 & $0.536-58.064$ \\
\hline Capillaria spp. & 1 & 7 & 0.893 & 0.344 & 0.398 & $0.052-3.030$ \\
\hline Raillietina spp & 0 & 1 & 0.894 & 0.344 & 0.000 & - \\
\hline Eimeria spp & 12 & 20 & 0.364 & 0.547 & 1.673 & $1.028-2.724$ \\
\hline Eim. + Capil. & 0 & 5 & 1.926 & 0.165 & 0.000 & - \\
\hline Total & 15 & 34 & & & & \\
\hline
\end{tabular}

Tabla 6. Análisis estadístico bivariado (Chi cuadrado) entre parásitos intestinales y comunas de Villavicencio.

\begin{tabular}{|c|c|c|c|c|c|c|c|}
\hline \multirow{2}{*}{ Parásito } & \multicolumn{3}{|c|}{ Comuna } & \multirow{2}{*}{$x^{2}$} & \multirow{2}{*}{$\mathbf{P}$} & \multirow{2}{*}{$\begin{array}{c}\text { Razón de } \\
\text { prevalencia }\end{array}$} & \multirow{2}{*}{$\begin{array}{c}\text { Intervalo de } \\
\text { confianza }\end{array}$} \\
\hline & 2 & 3 & 4 & & & & \\
\hline Ascaridia spp. & 3 & 0 & 0 & 1.993 & 0.369 & - & - \\
\hline Capillaria spp. & 5 & 3 & 0 & 2.788 & 0.248 & 1.760 & $0.476-6.496$ \\
\hline Raillietina spp. & 1 & 0 & 0 & 0.648 & 0.723 & - & - \\
\hline Eimeria spp. & 18 & 7 & 7 & 0.199 & 0.905 & 1.140 & $0.597-2.178$ \\
\hline Eim. + Capil. & 2 & 3 & 0 & 5.319 & 0.070 & 4.400 & $0.811-23.857$ \\
\hline Total & 29 & 13 & 7 & & & & \\
\hline
\end{tabular}

\section{DISCUSIÓN}

La paloma doméstica (Columba livia) se ha convertido en un serio problema de las grandes ciudades del mundo, dado el aumento descontrolado de su población; a esta problemática no escapa la ciudad de Villavicencio, en donde no existen programas oficiales de control, por lo que se pretendió identificar los endo y ectoparásitos en las palomas de tres comunas de la ciudad. Es conocido que algunos agentes infecciosos tienen importancia para la salud pública o para los sistemas de producción animal. Dentro de las más conocidas se encuentran la histoplasmosis, la enfermedad de Newcastle, psitacosis, criptococcosis, coccidiosis, toxoplasmosis, pseudotuberculosis y salmonelosis $(7,8,9,10)$.

La presencia de solo dos especies de ectoparásitos en las palomas de nuestro estudio fue bajo y menor si se compara con siete especies identificadas en palomas del mercado de San Martín de Porres en Lima - Perú (22), ocho especies en Irán (23) y 60 especies en otros estudios (24). Las cifras de prevalencia de ectoparasitosis en C. livia domestica pueden variar de acuerdo a varios factores como época del año, factores climáticos, eco-geográficos relacionados con rangos de los hospedadores $(25,26)$.

En la naturaleza, solo algunos de los artrópodos transporta patógenos, por lo que los huéspedes vertebrados son más a menudo expuesto a hospedadores libres de estos. Sin embargo, incluso si no se transmiten patógenos, la hematofagía causa irritación y distrae a los hospedadores, lo que a menudo desencadena defensas conductuales (como el acicalamiento) $(27,28)$. La literatura señala que Haemoproteus columbae y su vector la mosca Pseudolynchia canariensis tiene una distribución mundial, aunque con mayor presencia en la zona tropical (29). En nuestro estudio, el $26.54 \%$ de las palomas estaban infectadas con éste hemoparásito (Figura 7), prevalencia superior a la reportada en Zaria (Nigeria) del 15.2\% 
(30), similar a un estudio de Italia que reportó $29.4 \%$ (31) e inferior a los reportes de Sur Africa $96.9 \%$ (29) y $100 \%$ en Sao Paulo, Brazil (32). Es importante considerar que Haemoproteus columbae no ha sido reportado en hospedadores no columbidos y por lo tanto estos pueden ser sus hospedadores específicos (33). Posiblemente, la alta prevalencia de la infección dependa de la densidad de población de Haemoproteus spp. y su vector, así como la densidad de población de palomas ferales y sivestres, siendo generalmente altas en estudios suburbanos y urbanos $(32,34,35)$. Sin embargo, dado que las poblaciones naturales de $C$. livia han sido poco investigadas, no hay datos suficientes para comparar (29).

La distribución de los piojos (Columbicola columbae) en las plumas y cuerpo de las palomas, concuerda con Harbison y Boughton (36), quienes encontraron un mayor número de piojos adultos en abdomen, alas y cola, mientras que los piojos inmaduros se ubicaban en grandes cantidades en la cabeza y el cuello. La prevalencia de Columbicola columbae fue similar a la obtenida en Islas Canarias (37), aunque mayor a $82.8 \%$ reportado en Lima (38), $41.3 \%$ en Irán (39), $64 \%$ en Colombia (40) y $82 \%$ en Libia (41).

La prevalencia de ooquistes de Eimeria spp. en $65.3 \%$ de las muestras fue mayor a lo reportado en Islas Canarias de 50\% (37), 55\% en Envigado-Colombia (40) e inferior al 86.05\% reportado en Brazil (42), 55-89\% en Polonia (43) y el $90 \%$ reportado para la región de Montaña Verde en Libia (44). La coccidiosis suele cursar sin manifestaciones clínicas claras. Las palomas se ven saludables, pero son menos activas. En algunos casos observa una diarrea acuosa. En palomas jóvenes, la enfermedad es aguda. Las palomas enfermas presntan plumas erizadas y quebradizas están muertas y quebradizas, pérdida de peso y diarrea sanguinolenta. La mortalidad de los jóvenes oscila entre el 5\% y el $30 \%$. Además, en algunos casos se observa la inhibición del crecimiento y los trastornos del equilibrio (45). Muchos factores pueden contribuir a las diferencias en la prevalencia de coccidias entre las zonas y se destacan, lascondiciones climáticas, las prácticas agrícolas y las razas de palomas (46).

Dentro de los cestodos, el género Raillietina es considerado el más común en las palomas, en nuestro estudio se encontró un $2.0 \%$ de prevalencia, que es inferior al $7.8 \%$ reportado en
Venezuela(47) y al $44 \%$ del estudio de Foronda et al (37) en Nigeria, en donde el alto porcentaje fue atribuido a la época de muestreo (época lluviosa) cuando la actividad del parásito es más alta, ya que los huevos se desarrollan más rápido bajo condiciones de alta temperatura y humedad (48). En nuestro estudio, el muestreo se realizó en la época de transición verano-invierno en donde se tenía alta temperatura, pero baja humedad, lo que probablemente no favoreció la rápida eclosión de los huevos. Considerando que la dinámica de los diferentes parásitos puede cambiar bajo las condiciones ambientales no se debe menospreciar la importancia de este helminto, ya que las palomas pueden ser la fuente de infección para las aves de traspatio y explotaciones comerciales (21).

La baja presencia de huevos de nematodos observados por la técnica de flotación (Ascaridia $\mathrm{spp}=4.16 \%$ y Capillaria spp $=13.8 \%$ ), contrastan con las prevalencia reportadas en Libia del $22 \%$ (41), Brazil de $32.56 \%$ (42). Nuestro estudio reveló parasitismo gastrointestinal mixto, que concuerda con los estudios de Msoffe et al (49), Abed et al (50) y el de El-Dakhly et al (51), de igual forma Pérez-García et al (40) encontraron tres palomas con Capillaria spp y Ascaridia spp. mientras que cinco reportaron Capillaria spp y ooquistes de coccidios. La prevalencia de Capillaria sp. (16.3\%) fue mayor de lo observado de $4.32 \%$ por Kommu et al (52) y de $7.63 \%$ reportado en Nigeria (53).

La no diferencia en el parasitismo asociado al sexo encontrada en este estudio, coincide con estudios realizados en Turquía, en donde además se encontró que la estación del año tenía importancia (54). Aunque en Colombia dada su ubicación geográfica no se tienen estaciones, se podría especular que, en los períodos de sequía e invierno, las infestaciones de parásitos deberían ser distintas, conocimiento que debe ser abordado en futuros estudios. En igual sentido, las no diferencias en el parasitismo entre comunas sugiere condiciones epidemiológicas similares relacionadas con el hábitat, alimentación y contacto con otras aves.

Finalmente, los parásitos internos y externos reportados en otros estudios, se identificaron en las palomas de Villavicencio, aunque en menor magnitud. La realización de estudios longitudinales estaría indicados para conocer con mayor exactitud las mayores épocas de infestación parasitaria. Una estrategia de desparasitación masiva en el agua, podría ser 
Walteros-Casas et al - Parásitos en palomas domésticas (Columba livia) de Colombia

útil en la prevención de trasmisión de estos parásitos a la fauna nativa, a otros sistemas de producción y al humano, la anterior estrategia podría ser reforzada con la restricción del alimento en estas comunas de la ciudad, lo cual permitiría una disminución de la población.

\section{Conflicto de intereses}

Los autores declaran no tener conflicto de intereses.

\section{Agradecimientos}

Este trabajo fue financiado por la Universidad de Antioquia, UdeA, Medellín, Colombia (Estrategia de consolidación de Grupos de Investigación CODI 2018-2019)

\section{REFERENCIAS}

1. Donegan TM. The pigeon names Columba livia, 'C. domestica' and C. oenas and their type specimens. B Bri Ornithol Club. 2016; 136(1):14-27. https:// pigeonracingwebshop.com/content/ literature/breeding/The pigeon names.pdf

2. Del Hoyo J, Elliot A, Sargatal J. Handbook of the birds of the world. Vol. 4. Sandgrouse to Cuckoos. Lynx Ediciones: Barcelona, España; 1997.

3. Olalla A, Ruíz V, Ruvalcaba I, Mendoza R. Palomas, especies invasoras. CONABIO. Biodiversitas. 2009; 82:7-10. https:// fumigacionesbw.com/documentos/pdf/ Palomas\%20especie\%20invasora

4. Baptiste MP, Castaño N, Cárdenas-López D, Gutiérrez FP, GIL DL, LASSO CA. Análisis de riesgo y propuesta de categorización de especies introducidas para Colombia. Instituto de Investigación de Recursos Biológicos Alexander von Humboldt: Bogotá, D.C., Colombia; 2010. http:// repository.humboldt.org.co/bitstream/ handle/20.500.11761/31384/191. pdf?sequence $=1$ \&isAllowed $=y$

5. Spennemann DHR, Pike M, Watson MJ. Behaviour of Pigeon Excreta on Masonry Surfaces. Rest of Build and Monum 2017; 23(1):15-28. https://doi.org/10.1515/rbm2017-0004

6. Mattews S. Programa Mundial sobre Especies Invasoras. Primera edición. Secretaría del GISP. 2005. http://www. issg.org/pdf/publications/gisp/resources/ samericainvaded-es.pdf
7. Cano-Terriza D, Guerra R, Lecollinet S, Cerdgravea-Cuellar M, Cabezón O, Almería S, Garcia-Bocanegra I. Epidemiological survey of zoonotic pathogens in feral pigeons (Columba livia var. domestica) and sympatric zoo species in Southern Spain. Comp Immunol Microbiol Infect Dis. 2015; 43:22-7. https://doi.org/10.1016/j. cimid.2015.10.003

8. Méndez-Mancera VM, Villamil-Jiménez LC, Buitrago-Medina DA, Soler-Tovar D. La paloma (Columba livia) en la transmisión de enfermedades de importancia en salud pública. Revista Ciencia Animal. 2013; 1(6):177-194. https://ciencia.lasalle.edu. $\mathrm{co} / \mathrm{ca} / \mathrm{vol} / \mathrm{iss} 6 / 13 /$

9. Ghaderi Z, Eidi S, Razmyar J. High Prevalence of Cryptococcus neoformans and Isolation of Other Opportunistic Fungi from Pigeon (Columba livia) Droppings in Northeast Iran. J Avian Med Surg. 2019; 33(4):335-339. https://doi.org/10.1647/2018-370

10. Vázquez B, Esperón F, Neves E, López J, Ballesteros C, Muñoz MJ. Screening for several potential pathogens in feral pigeons (Columba livia) in Madrid. Acta Vet Scand. 2010; 52:45. https://doi. org/10.1186/1751-0147-52-45

11. Ghosh KK, Islam MS, Sikder S, Das S, Chowdhury $S$ and Alim MA. Prevalence of ecto and gastrointestinal parasitic infections of pigeon at Chittagong metropolitan area, Bangladesh. J Adv Parasitol. 2014; 1(1):911. https://doi.org/10.14737/journal. jap/2014/1.1.9.11 
12. Adang $\mathrm{KL}$, Oniye $\mathrm{SJ}$, Ezealor $\mathrm{AU}$ Abdu $\mathrm{PA}$, Ajanusi OJ, Yoriyo KP. Ectoparasites and intestinal helminths of speckled pigeon (Columba guinea Hartlaub and Finsch 1870) in Zaria, Nigeria. Science World Journal. 2009; 4:2. https://www.ajol.info/index. $\mathrm{php/swj/article/view/51836}$

13. Radfar $\mathbf{M H}$, Khedri J, Adinehbeigi M, Nabavi $\mathrm{R}$, Rahmani R. Prevalence of parasites and associated risk factors in domestic pgeons (Columba livia domestica) and free-range backyard chickens of Sistan region, east of Iran. J Parasit Dis. 2012; 36(2):220-225. https://doi.org/10.1007/s12639-012-0112-5

14. Villalba-Sánchez C, De La Ossa-Lacayo A, De La Ossa J. Densidad de paloma doméstica (Columbia livia doméstica Gmelin, 1789) en el nuevo mercado público de Sincelejo, Sucre, Colombia. Rev UDCA Act \& Div Cient. 2015; 18(2):497-502. https://doi. org/10.31910/rudca.v18.n2.2015.265

15. Shimaa MG, Mansour RM. ElBakrey HA, Knudsen DE Amal AME. Natural infection with highly pathogenic avian influenza virus H5N1 in domestic pigeons (Columba livia) in Egypt, Avian Path. 2014; 43(4):319-324. https://doi.org/10.1080/03079457.2014.9 $\underline{26002}$

16. Clayton D, Price R. Taxonomy of the New World Columbicola (Phthiraptera: Philopteridae) from the Columbiformes (Aves), with descriptions of five new species. Ann Entom Soc Amer. 1999; 92(5):675-685. https://doi.org/10.1093/aesa/92.5.675

17. Atkinson $\mathrm{CT}$, Hunter B. Parasitic diseases of wild birds. New Jersey, USA: WileyBlackwell; 2008.

18. Graciolli G, Carvalho CJB. Hippoboscidae (Diptera, Hippoboscidae) no Estado do Paraná, Brasil: chaves de identificação, hospedeiros e distribuição geográfica. Rev Bra de Zool 2003; 20(4):667-674. https://doi. org/10.1590/S0101-81752003000400019

19. Becker AC, Kraemer A, Epe C, Strube C. Sensitivity and efficiency of selected coproscopical methods - sedimentation, combined zinc sulfate sedimentation-flotation, and McMaster method. Parasit Res. 2016; 115: 2581-2587. https://doi.org/10.1007/ $\underline{\text { s00436-016-5003-8 }}$
20. Hermosilla C, Barbisch B, Heise A, Kowalik $\mathrm{S}$, Zahner H. Development of Eimeria bovis in vitro: suitability of several bovine, human and porcine endothelial cell lines, bovine fetal gastrointestinal, Madin-Darby Bovine Kidney (MDBK) and African green monkey kidney (VERO) cells. Parasitol Res. 2002; 88:301. https://doi.org/10.1007/s00436001-0531-1

21. Soulsby EJL. Helminths, Arthropods and Protozoa of Domesticated Animals. 7 7th Edn. Bailliere and Tindall: London, UK; 1986.

22. Naupay A, Castro J, Caro J, Sevilla L, Hermosilla J, Larraín K. Ectoparásitos en palomas Columba livia comercializadas en un mercado del distrito de San Martín de Porres, Lima, Perú. Rev Invest Vet Perú. 2015; 26(2):259-265. https://dx.doi. org/10.15381/rivep.v26i2.11094

23. Chaechi-Nosrati M, Eslami A, Rahbari S. The survey of parasitic infections of wild pigeons (Columba livia) in Lahijan city, Guilan, Iran. Comp Clinical Pathol. 2018; 27:1405. https:// doi.org/10.1007/s00580-018-2779-1

24. De La Ossa J, De La Ossa-Lacayo A, MonroyPineda M. Abundancia de paloma doméstica (Columbia livia domestica Gmelin, 1789) en Santiago de Tolú, Sucre, Colombia. Rev MVZ Córdoba. 2017; 22(1):5718-5727. https:// dx.doi.org/10.21897/rmvz.931

25. Adang KL, Oniye SJ, Ezealor AU, Abdu PA, Ajanusi OJ. Ectoparasites of Domestic Pigeon (Columba livia domestica, Linnaeus) in Zaria, Nigeria. Research J Parasitol. 2008; 3(2):79-84 https://doi.org/10.3923/ jp. 2008.79.84

26. Begum A, Sehrin S. Prevalence and seasonal variation of ectoparasite in pigeon, Columba livia (Gmelin, 1789) of Dhaka, Bangladesh. Bangladesh J Zool 2011; 39(2):223-230. https://doi.org/10.3329/BJZ.V39I2.10592

27. Koop JAH, Huber SK, Clayton DH: Does sunlight enhance the effectiveness of avian preening for ectoparasite control? J Parasitol 2012; 98(1):46-48. https://doi. org/10.1645/GE-2889.1 
28. Clayton DH, Tompkins DM. Comparative effects of mites and lice on the reproductive success of rock doves (Columba livia). Parasitol. 1995; 110(2):195-206. https:// doi.org/10.1017/s0031182000063964

29. Nebel C, Harl J, Pajot A, Weissenböck $H$, Amar A, Sumasgutner P. High prevalence and genetic diversity of Haemoproteus columbae (Haemosporida: Haemoproteidae) in feral pigeons Columba livia in Cape Town, South Africa. Parasitol Res. 2020; 119:447463. https://doi.org/10.1007/s00436-019$\underline{06558-6}$

30. Natala AJ, Asemadahun ND, Okubanjo OO, Ulayi BM, Owolabi YH, Jato ID and YusufKH. A Survey of Parasites of Domesticated Pigeon (Columba livia domestic) in Zaria, Nigeria. Intern J of Soft Comput. 2009; 4:148-150. https://medwelljournals.com/ abstract/?doi=ijscomp.2009.148.150

31. Scaglione F, Pregel P, Tiziana F, Pérez A, Ferroglio E, Bollo E. Prevalence of new and known species of haemoparasites in feral pigeons in northwest Italy. Malaria J. 2015; 14:99. https://doi.org/10.1186/s12936$\underline{015-0617-3}$

32. Chagas $C R F$, Guimarães $L$ de O, Monteiro EF, Valkiūnas G, Katayama MV, Santos SV, Kirchgatter K. Hemosporidian parasites of free-living birds in the São Paulo Zoo, Brazil. Parasit Research. 2015; 115(4):1443-1452. https://doi.org/10.1007/s00436-015-4878-0

33. Valkiunas G, Zickus T, Shapoval AP, Lezhova TA. Effect of Haemoproteus belopolskyi (Haemosporida: Haemoproteidae) on body mass of the blackcap Sylvia atricapilla. J Parasitol. 2006; 92(5):1123-1125. https:// doi.org/10.1645/GE-3564-RN.1

34. Karamba KI, Kawo AH, Dabo NT, Mukhtar MD. A survey of avian malaria parasite in Kano State, Northern Nigeria. Int J Biotechnol Mol Biol Res. 2012; 3(1):8-14. https://academicjournals.org/journal/ IJBMBR/article-full-text-pdf/C0854E22188

35. Sol D, Jovani R, Torres J. Geographical variation in blood parasites in feral pigeons: the role of vectors. Ecography. 2000; 23(3):307-314. https://doi. org/10.1111/j.1600-0587.2000.tb00286.x
36. Harbison CW, Boughton RM. Thermoorientation and the movement of feather-feeding lice on hosts. J Parasitol. 2014; 100(4):433-441. https://doi. org/10.1645/13-374.1

37. Foronda $P$, Valladares $B$, Rivera-Medina JA, Figueruelo E, Abreu N, Casanova JC. 2004. Parasites of Columba livia (Aves: Columbiformes) in Tenerife (Canary Islands) and their role in the conservation biology of the Laurel pigeons. Parasite. 2001; 11(3):311-316. https://doi.org/10.1051/ parasite/2004113311

38. Balicka-Ramisz A, Pilarczyk P. Occurrence of coccidia infection in pigeons in amateur husbandry. Diagnosis and prevention. Original papers. Ann Parasitol. 2014; 60(2):93-97. https://pubmed.ncbi.nlm.nih. gov/25115060/

39. Radfar $\mathrm{MH}$, Khedri J, Adinehbeigi K, Nabavi $\mathrm{R}$, Rahmani K. Prevalence of parasites and associated risk factors in domestic pigeons (Columba livia domestic) and free-range backyard chickens of Sistan region, east of Iran. J Parasitol Dis. 2012; 36(2):220-225. https://pubmed.ncbi.nlm. nih.gov/24082532

40. Pérez-García J, Monsalve-Arcila D, Márquez-Villegas. Presencia de parásitos y enterobacterias en palomas ferales (Columba livia) en áreas urbanas en Envigado, Colombia. Rev Fac Nac Salud Pública. 2015; 33(3):370-376. https://doi. org/10.17533/udea.rfnsp.v33n3a06

41. Alkharigy FA, El Naas AS, El Maghrbi AA. Survey of parasites in domestic pigeons (Columba livia) in Tripoli, Libya. Open Vet J. 2018; 8(4):360-366. http://dx.doi. org/10.4314/ovj.v8i4.2

42. Tietz-Marques S, De Quadros R, Da Silva C, Baldo M. . Parasites of pigeons (Columba livia) in urban areas of Lages, Southern Brazil. Parasitol Latinoam. 2007; 62(34):183-187. https://doi.org/10.4067/ $\underline{\mathrm{s} 0717-77122007000200014}$

43. Balicka-Ramisz A, Bogumiła P. Occurrence of coccidia infection in pigeons in amateur husbandry. Diagnosis and prevention. Ann Parasitol. 2014; 60(2):93-97. https:// pubmed.ncbi.nlm.nih.gov/25115060/ 
44. Eljadar M, Saad W, Elfadel G. 2012. A study on the prevalence of endoparasites of domestic Pigeons (Columba livia domestica) inhabiting in the Green Mountain Region of Libya. J Am Sci. 2012; 8(12):191193. http://www.dx.doi.org/10.7537/ marsjas081212.28

45. Krautwald-Junghanns ME, Zebisch R, Schmidt V. Relevance and treatment of coccidiosis in domestic pigeons (Columba livia forma domestica) with particular emphasis on toltrazuril. J Avian Med Surg. 2009; 23:1-5. https://doi.org/10.1647/2007-049R.1

46. Dong $\mathrm{H}$, Zhao Q, Zhu S, Han $\mathrm{H}$, Huang B. Prevalence of Eimeria infection in domestic pigeons (Columba livia domestica) in Shanghai, China. J Vet Med Res. 2018; 5(8):1155. https://www. jscimedcentral.com/VeterinaryMedicine/ veterinarymedicine-5-1155.pdf

47. Cazorla-Perfetti D, Morales-Moreno. Parásitos intestinales en poblaciones ferales de palomas domésticas (Columba livia domestica) en Coro, estado Falcón, Venezuela. Rev Inv Vet Perú. 2019; 30(2):836-847. http://dx.doi. org/10.15381/rivep.v20i2.16214

48. Ademola IO, Fagbohun OA. Helminth parasites of domestic pigeons (Columba livia) in Ibadan, Nigeria. Bull Anim Health Prod Afri. 2005; 53(2):147-148. https://doi. org/10.4314/bahpa.v53i2.32703

49. Msoffe PLM, Muhairwa AP, Chiwanga $\mathrm{GH}$, Kassuku AA. A study of ecto- and endoparasites of domestic pigeons in Morogoro Municipality, Tanzania. Afr J Agric Res. 2010; 5(3):264-267. https://doi. org/10.5897/AJAR09.451
50. Abed $A A, N a j i ~ H A$, Rhyaf AG. Investigation study of some parasites infected domestic pigeon (Columba livia domestic) in $\mathrm{Al}$ Dewaniya. IOSR- J Pharm Biol Sci. 2014; 9(4):13-20. http://www.iosrjournals.org/ iosr-jpbs/papers/Vol9-issue4/Version-4/ C09441320.pdf

51. El-Dakhly KM, Mahrous LN, Mabrouk GA. Distribution pattern of intestinal helminths in domestic pigeons (Columba livia domestica) and turkeys (Meleagris gallopavo) in Beni-Suef province, Egypt. J Vet Med Res. 2016; 23(1):112-120. https:// dx.doi.org/10.21608/jvmr.2016.43226

52. Kommu S, Rajeshwari G, Sreenivasamurthy G.S. Prevalence of helminthic and protozoan infections in pigeons- in and around Hyderabad of Telangana state. J Parasit Dis Diagn Ther. 2016; 1(1):1-3. http://www. alliedacademies.org/parasitic-diseasesdiagnosis-therapy/

53. Balarabe RM, Malang KS, Rowland IS, Auwalu HA. Detection of Capillaria obsignata of Pigeons (Columba livia domestica) from Kano State, Nigeria. Res J Parasitol. 2017; 12(2):45-49. https://doi.org/10.3923/ jp.2017.45.49

54. Senlik B, Gulegen E, Akyol V. Effect of age, sex and season on the prevalence and intensity of helminth infections in domestic pigeons (Columba livia) from Bursa Province, Turkey. Acta Vet Hung. 2005; 53:449-456. https://doi.org/10.1556/AVet.53.2005.4.5 